\title{
Cascaded Residual Dense Networks for Dynamic MR Imaging with Edge-Enhanced Loss Constraint
}

Investigative Magnetic
Resonance
Imaging

\section{Original Article}

\section{Received: June 4, 2020}

Revised: September 8, 2020

Accepted: November 10, 2020

\section{Correspondence to:}

Dong Liang, Ph.D.

Shenzhen Institutes of Advanced Technology, Chinese Academy of Sciences, China.

Tel. +0755-86392243

Fax. +0755-86392299

E-mail: dong.liang@siat.ac.cn

This is an Open Access article distributed under the terms of the Creative Commons Attribution Non-Commercial License (http://creativecommons.org/licenses/ by-nc/4.0/) which permits unrestricted non-commercial use, distribution, and reproduction in any medium, provided the original work is properly cited.

Copyright (C) 2020 Korean Society of Magnetic Resonance in Medicine (KSMRM)

\author{
Ziwen Ke ${ }^{1,2}$, Yanjie Zhu' ${ }^{1}$, Dong Liang ${ }^{1}$ \\ ${ }^{1}$ Paul C. Lauterbur Research Center for Biomedical Imaging, Shenzhen Institutes of Advanced \\ Technology, Chinese Academy of Sciences, Shenzhen, China \\ ${ }^{2}$ Shenzhen College of Advanced Technology, University of Chinese Academy of Sciences, Shenzhen, \\ China
}

Dynamic magnetic resonance (MR) imaging has generated great research interest, because it can provide both spatial and temporal information for clinical diagnosis. However, slow imaging speed or long scanning time is still a challenge for dynamic $M R$ imaging. Most existing methods reconstruct dynamic MR images from incomplete $k$-space data under the guidance of compressed sensing (CS) or lowrank theory, which suffer from long iterative reconstruction time. Recently, deep learning has shown great potential in accelerating dynamic MR. Our previous work proposed a dynamic MR imaging method with both $k$-space and spatial prior knowledge integrated via multi-supervised network training. Nevertheless, there was still some smoothing needed in the reconstructed images at high acceleration. In this work, we propose cascaded residual dense networks for dynamic MR imaging with edge-enhanced loss constraint, dubbed cascaded residual dense networks (CRDN). Specifically, the cascaded residual dense networks fully exploit the hierarchical features from all the convolutional layers with both local and global feature fusion. We further use the higher-degree total variation loss function, which has the edge enhancement properties, for training the networks.

Keywords: Dynamic MR imaging; Deep learning; Compressed sensing; Dense; Local feature; Global feature; Total variation

\section{INTRODUCTION}

Dynamic MR imaging is a non-invasive imaging technique that can provide both spatial and temporal information for the underlying anatomy. Nevertheless, both physiological and hardware constraints have made it suffer from slow imaging speed or long imaging time, which may lead to patients' discomfort or sometimes cause severe motion artifacts. Therefore, it is necessary to accelerate MR imaging.

To accelerate a dynamic MR scan, there have been three types of efforts, namely, in developing physics-based fast imaging sequences (1), hardware-based parallel imaging techniques (2), and MR image reconstruction based on signal processing of incomplete $k$-space data. Our specific focus here is undersampled MR image reconstruction, which requires prior information to resolve the aliasing artifacts caused by the violation of the 
Nyquist sampling theorem. Specifically, the reconstruction task is normally formulated as solving an optimization problem with two terms, which are data fidelity and prior regularization. Popular prior information includes sparsity, which prompts the image to be sparsely represented in a certain transform domain while being reconstructed from incoherently undersampled $k$-space data. These techniques are well known as CS-MRI $(3,4)$. For example, $k$ - $t$ FOCUSS $(5)$ takes advantage of the sparsity of $x-f$ support to reconstruct $x-f$ images from the undersampled $k-t$ space. It encompasses the celebrated $k$ - $t$ BLAST and $k$ - $t$ SENSE (6) as special cases. And $k-t$ ISD (7) incorporates additional information on the support of the dynamic image in $x-f$ space based on the theory of CS with partially known support. DLTG (8) can learn redundancy in the data via an auxiliary constraint on temporal gradients (TG) sparsity. Wang and Ying (9) employ a patch-based 3-D spatiotemporal dictionary for sparse representations of dynamic image sequences. Besides, low rank is also a prior regularization. It can use low-rank and incoherence conditions to complete missing or corrupted entries of a matrix. A typical example of low rank is $L+S$ (10), where the nuclear norm is used to enforce low rank in $L$, and the $L 1$ norm is used to enforce sparsity in $S$. And $k$ - $t$ SLR (11) exploits the correlations in the dynamic imaging dataset by modeling the data to have a compact representation in the Karhunen Louve transform (KLT) domain. These methods greatly improved the spatiotemporal resolution of dynamic MR imaging; however, their iterative solution procedures require a rather long time to achieve high-quality reconstructions, and regularization parameter selection is empirical. Additionally, most of these CS-based approaches exploit prior information from only the to-bereconstructed images or from only a few reference images: prior knowledge from big data is not used.

On the other hand, deep learning has shown great potential in accelerating MR imaging. There have been several newly proposed methods, which can be roughly categorized into two types: model-based unrolling methods (12-14) and end-to-end learning methods (1523). The model-based unrolling methods adapt the iterative procedure of traditional optimization algorithms to network learning. They adaptively learn all the parameters of regularization terms and transforms in the model by network training. For example, in VN-Net (13), generalized compressed sensing reconstruction formulated as a variational model is embedded in an unrolled gradient descent scheme. ADMM-Net (12) is defined over a data flow graph, which is derived from the iterative procedures in Alternating Direction Method of Multipliers (ADMM) algorithm for optimizing a CS-based MRI model. The other type uses the big-data information to learn a network that maps between the undersampled and fully sampled data pairs. Wang et al. (15) train a deep neural network to learn the mapping relationship between undersampled and fully sampled brain MR images. AUTOMAP (18) learns a mapping between the sensor and the image domain from appropriate training data. Despite all these successes, there are only three studies that specifically apply to dynamic MR imaging (22-24). A deep cascaded convolutional neural network (DC-CNN) is designed in (22), and a convolutional recurrent neural network (CRNN) is proposed in (24). Both works make great contributions to dynamic MR imaging. Nevertheless, the reconstruction results can still be improved, given two observations. First, they have adopted a single supervised loss function that considers only the fidelity between the final output and the ground truth. The intermediate results have not been used for the supervision. Furthermore, all their networks are built in the spatial domain where only data-consistency layers are considered for the direct $k$-space correction and no other $k$-space information is used. So in our other work, we proposed a dynamic MR imaging method with both $k$-space and spatial prior knowledge integrated via multi-supervised network training, dubbed DIMENSION (23). Although the DIMENSION model improved the reconstruction results for dynamic MR imaging more than did other methods, there is still a certain degree of smoothing needed in the reconstructed images at high acceleration factors, perhaps because the loss function used in (23) is MSE. The MSE loss functions indicate only the mean square information between the reconstructed image and the ground truth and cannot perceive the image structural information. Furthermore, none of the three peer-reviewed works (DC-CNN, CRNN, and DIMENSION) make full use of the hierarchical features from the original $k$-space data, thereby achieving relatively low performance.

In this work, we propose cascaded residual dense networks for dynamic MR imaging with edge-enhanced loss constraint, dubbed CRDN. The improvements are mainly reflected in the residual dense network structures and the total variation loss function. Our contributions can be summarized as follows:

1. In this work, we propose cascaded residual dense networks for dynamic MR imaging, which can fully exploit the hierarchical features from all the convolutional layers with both local and global feature fusion.

2. We further use the total variation (TV) loss function, 
which has the edge-enhancement properties, for training the networks. We explore the influence of different types of TV constraints on dynamic MR reconstruction, including isotropic TV, anisotropic TV and higher-degree total variation (HDTV). We find that using the TV constraint improved the reconstruction quantitatively, among which anisotropic TV worked best.

\section{MATERIALS AND METHODS}

\section{METHODOLOGY}

\section{CS-MRI and CNN-MRI}

According to CS (4), MR images with a sparse representation in some transform domain can be reconstructed from randomly undersampled $k$-space data. Let $S \in C^{N_{x} N_{y} N_{t}}$ represent a complex-valued dynamic MR image. The problem can be described by the following formula:

$$
K_{\mathrm{u}}=F_{\mathrm{u}} S+\mathrm{e}
$$

where $K_{u} \in C^{N_{x} N_{y} N_{t}}$ is the undersampled measurements in $k$-space and the unsampled points are filled with zeros. $F_{u}$ is an undersampled Fourier encoding matrix, and $e \in$ $C^{N_{x} N_{y} N_{t}}$ is the acquisition noise. We want to reconstruct $S$ by solving the inverse problem of Eq. [1]. However, the inverse problem is ill-posed; hence the reconstruction is not unique. In order to reconstruct $S$, we constrain this inverse problem by adding some prior knowledge and solve the following optimization problem:

$$
\min _{S} \frac{1}{2}\left\|F_{\mathrm{u}} S-K_{\mathrm{u}}\right\|_{2}^{2}+\lambda R(S)
$$

The first term is the data fidelity, which ensures that the undersampled $k$-space of reconstruction is consistent with the actual measurements in k-space. The second term is often referred to as a priori regularization. In the methods of $C S, R(S)$ is usually a sparse prior of $S$ in some transform domains, e.g., finite difference, wavelet transform, or discrete cosine transformation.

In CNN-based methods, $R(S)$ is a CNN prior of $S$, which forces $S$ to match the output of the networks:

$$
\min _{S} \frac{1}{2}\left\|F_{\mathrm{u}} S-K_{\mathrm{u}}\right\|_{2}^{2}+\lambda\left\|S-f_{C N N}\left(\mathrm{~S}_{\mathrm{u}} \mid \theta\right)\right\|_{2}^{2}
$$

where $S_{u}$ is the undersampled image and $f_{C N N}\left(S_{u} \mid \theta\right)$ is the output of the CNN under the parameters $\theta$. The training process of the networks is to find the optimal parameters $\theta^{*}$. Once the networks are trained, the networks' output $f_{C N N}$ $\left(S_{u} \mid \theta^{*}\right)$ is the reconstruction we want.

\section{The Proposed Method}

\section{a. The Proposed CRDN}

In this work, we propose CRDN for cardiac MR image reconstruction (shown in Fig. 1). In this work, we still adopt the idea of cross-domain learning in the DIMENSION method (23). The network framework includes $k$-space prediction networks (KPN) and CRDN in a spatial domain. The two parts are connected by a Fourier inversion (see Inverse Fast Fourier Transform [IFFT] in Fig. 1). The details of the KPN can be found in (23). Here, we will focus on CRDN.

The CRDN consists of a CRDN. Each RDN contains five major components: shallow feature extraction, residual dense blocks (RDBs), global feature fusion, global residual learning, and data consistency (DC). First, the cardiac MR images predicted by the KPN are fed into the network for shallow feature extraction. Second, the shallow features go through $D$ RDBs for local feature fusion. The details of one RDB are shown in Fig. 2. The RDB includes dense connections, local feature fusion, and local residual connections. Dense connections refer to the direct connections of each convolutional layer to subsequent layers, which can improve the transmission of local features. All local features are concatenated together and pass through a $1^{*} 1$ convolutional layer to achieve local feature fusion. Residual connections [27] are introduced in RDB to further improve information propagation. Third, these residual dense features from $D$ RDBs are merged via global feature fusion (concatenation $+1^{*} 1$ convolution). We believe the combination of local feature fusion and global feature fusion can make full use of features at different levels. Fourth, a global residual connection combines the shallow features with the global fused features. Finally, a data consistency layer (22) is appended to correct MR images by the accurate $k$-space samples.

Compared with the published deep-learning-based dynamic MR methods, which used only plain CNNs and made the reconstruction results more dependent on the deeper features, the proposed CRDN method can fuse local and global features, which can not only avoid the gradient dispersion problem, but also make full use of hierarchical features. 


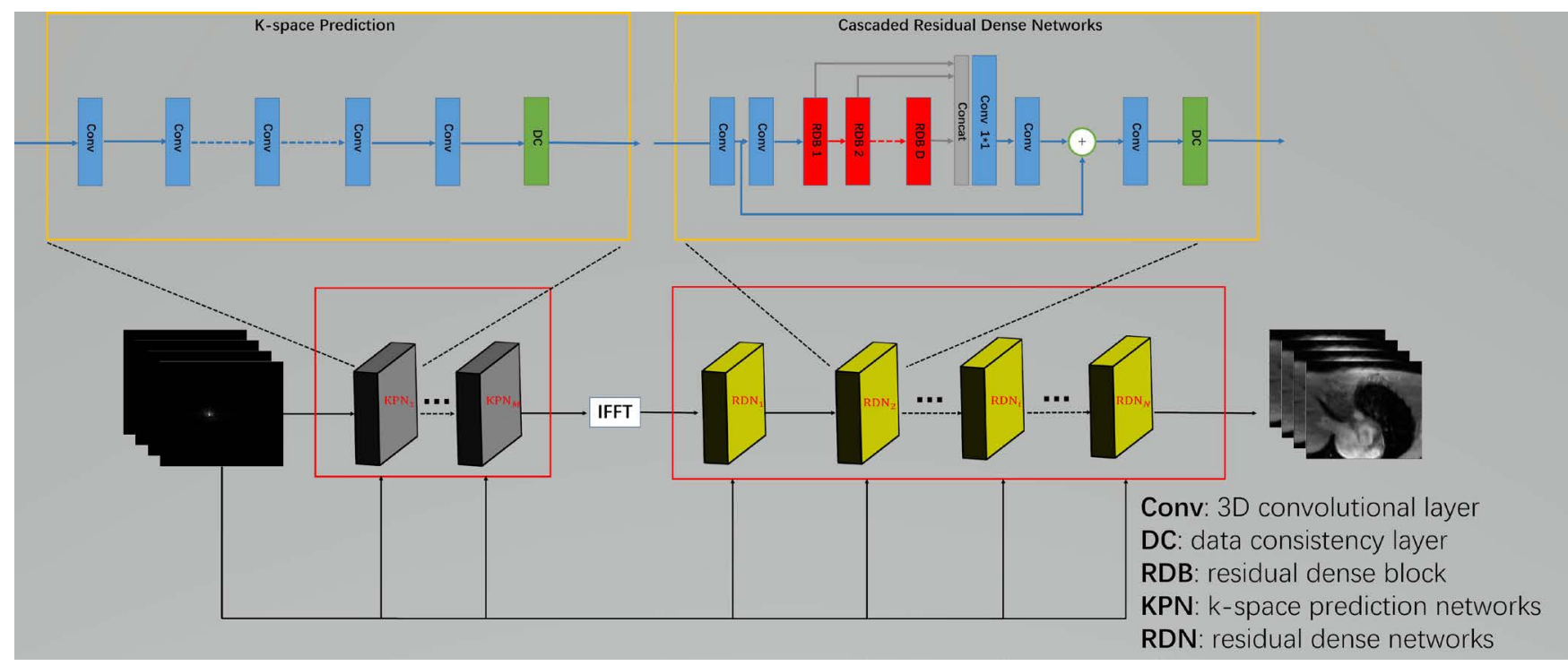

Fig. 1. The cascaded residual dense networks for dynamic MR imaging. The CRDN consists of a cascade of residual dense networks (RDN). Each RDN contains five major components: shallow feature extraction, residual dense blocks (RDBs), global feature fusion, global residual learning, and data consistency (DC).

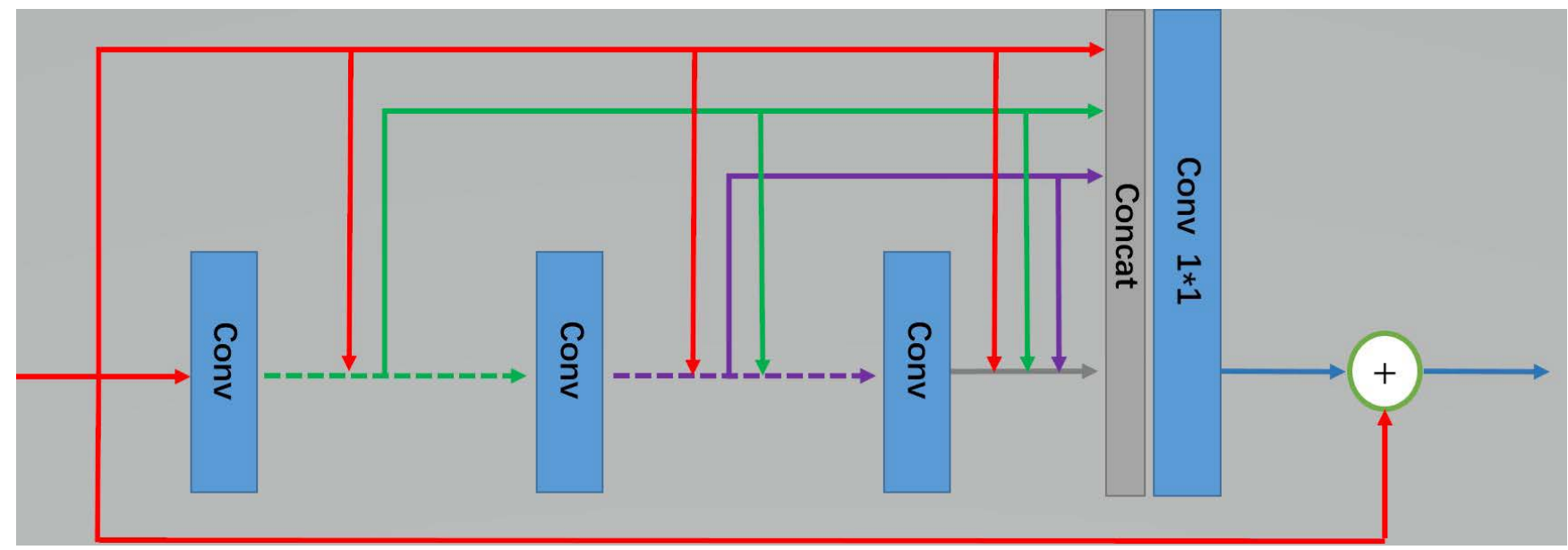

Fig. 2. Residual dense block (RDB) for local feature fusion.

\section{b. The Proposed Loss Function}

CNN-based methods (22-24) can achieve improved reconstruction results in less time than is needed by classical CS or low-rank-based methods (7-11). However, some smoothing is still needed in the reconstructed images at high acceleration factors, perhaps partly because of the loss functions, MSE, used in these works. The MSE loss functions indicate only the mean square information between the reconstructed image and the ground truth and cannot perceive the image structure information. In this work, we explore the effects of different TV constraints on network training, including anisotropic TV, isotropic TV, and
HDTV (25).

Let $\hat{S}$ be the output of the CRDN. Then the anisotropic TV can be defined as:

$$
\operatorname{TV}_{\text {aniso }}(\hat{S})=\int_{\Omega}\left|\frac{\partial \hat{S}(r)}{\partial x}\right|+\left|\frac{\partial \hat{S}(r)}{\partial y}\right| d r
$$

and the isotropic TV can be defined as:

$$
\mathrm{TV}_{\text {iso }}(\hat{\mathrm{S}})=\int_{\Omega} \sqrt{\left(\frac{\partial \hat{S}(r)}{\partial \mathrm{x}}\right)^{2}+\left(\frac{\partial \hat{S}(r)}{\partial y}\right)^{2}} d r
$$


The derivation and proof of HDTV can be seen in (25). Here, we directly give out the 2-degree TV (2DTV) and 3-degree TV (3DTV):

$$
\begin{aligned}
& 2 \mathrm{DTV}(\hat{S})=\int \sqrt{3\left(\left|\hat{S}_{x x}\right|^{2}+3\left|\hat{S}_{y y}\right|^{2}+4\left|\hat{S}_{x y}\right|^{2}+2 \mathrm{R}\left(\hat{S}_{x x} \hat{S}_{y y}\right)\right) / 8} \\
& 3 D T V(\hat{S})=\int \sqrt{5\left(\left|\hat{S}_{x x}\right|^{2}+\left|\hat{S}_{y y}\right|^{2}\right)+6 R\left(\hat{S}_{x x x} \hat{S}_{x y y}+\hat{S}_{y y y} \hat{S}_{x x y}\right)+} \\
& \left.\overline{9\left(\left|\hat{\mathrm{S}}_{x x y}\right|^{2}+\left.\hat{\mathrm{S}}_{x y y}\right|^{2}\right.}\right) d r / 4 \sqrt{2}
\end{aligned}
$$

\section{EXPERIMENTAL RESULTS}

\section{Setup}

\section{a. Data Acquisition}

We collected 101 fully sampled cardiac MR datasets using a 3T scanner (SIEMENS MAGNETOM Trio, Erlangen, Germany) with a T1-weighted FLASH sequence. We obtained written informed consent from all human subjects. Each scan contains a single slice FLASH acquisition with 25 temporal frames. The following parameters were used for FLASH scans: $F O V=330 \times 330 \mathrm{~mm}$, acquisition matrix 192 $\times 192$, slice thickness $=6 \mathrm{~mm}, \mathrm{TR}=3 \mathrm{~ms}, \mathrm{TE}=50 \mathrm{~ms}$, and 24 receiving coils. The raw multi-coil data of each frame was combined by an adaptive coil combining method (26) to produce a single-channel complex-valued image. We randomly selected $90 \%$ of the entire dataset for training and $10 \%$ for testing. Deep learning has a high demand for data volume (28). Therefore, we applied some data augmentation strategies. We shear the original images along the $x, y$ and $t$ directions. The sheared size is $117 x$ $120 \times 6(x \times y \times t)$, and the stride along the three directions is 7,7 , and 5 , respectively. Finally, we obtained $175003 \mathrm{D}$ complex-valued cardiac MR data with the size of $117 \times 120$ $\times 6$.

For each frame, the original $k$-space was retrospectively undersampled with 6 ACS lines. Specifically, we fully sampled frequency-encodes (along $k_{x}$ ) and randomly undersampled the phase encodes (along $k_{y}$ ) according to a zero-mean Gaussian variable-density function (5).

\section{b. Network Training}

All the CNN-based methods keep the same hyperparameters. For network training, we divided each dataset into two channels, where the channels store real and imaginary parts of the data. So the inputs of the network are undersampled $k$-spaces $R^{2 N_{x} N_{y} N_{t}}$ and the outputs are reconstructed images $R^{2 N_{x} N_{y} N_{t}}$. In this work, we focus on a D5C5 model, which works well for the DC-CNN model. The D5C5 model consists of 5 blocks (C5), each of which has 5 convolutional layers (D5). In order to simplify the parameters $\theta$ and make a fair comparison with the $\mathrm{D} 5 \mathrm{C} 5$ model, the KPN contains one frequency domain block, and the CRDN consists of four blocks, each of which contains five convolutional layers. Therefore, both the proposed model and the D5C5 model have 25 convolutional layers in total. He initialization (27) was used to initialize the network weights. Rectifier Linear Units (ReLU) (29) were selected as the nonlinear activation functions. The minibatch size was 20 . The exponential-decay learning rate (31) was used in all CNN-based experiments, and the initial learning rate was set to 0.0001 with a decay of 0.95 . All the models were trained by the Adam optimizer (32) with parameters $\beta_{1}=0.9, \beta_{2}=0.999$, and $\varepsilon=10^{-8}$.

The models were implemented on an Ubuntu 16.04 LTS (64-bit) operating system equipped with an Intel Xeon E52640 Central Processing Unit (CPU) and Tesla TITAN Xp Graphics Processing Unit (GPU, 12 GB memory) in the open framework Tensorflow (33) with CUDA and CUDNN support.

\section{c. Performance Evaluation}

For a quantitative evaluation, mean square error (MSE), peak signal-to-noise ratio (PSNR), and structural similarity index (SSIM) (34) were measured as follows:

$$
\begin{aligned}
& \text { MSE }=\| \text { Ref-Rec } \|_{2}^{2} \\
& \text { PSNR }=20 \log _{10} \frac{\max (\operatorname{Ref}) \sqrt{\mathrm{N}}}{\|\operatorname{Ref}-\operatorname{Rec}\|_{2}} \\
& \mathrm{SSIM}=\mid(\operatorname{Ref}, \operatorname{Rec}) \cdot c(\operatorname{Ref}, \operatorname{Rec}) \cdot s(\operatorname{Ref}, \operatorname{Rec})
\end{aligned}
$$

where $R e c$ is the reconstructed image, Ref denotes the reference image, and $N$ is the total number of image pixels. The SSIM index is a multiplicative combination of the luminance term, the contrast term, and the structural term (details shown in (34)).

\section{Experimental Design}

\section{a. Does the CRDN Work?}

To demonstrate the efficacy of the CRDN, we compared CRDN with a classical CS/LR method L+S (10) and two state-of-the-art deep-learning methods, including the D5C5 and DIMENSION methods at 4-fold acceleration, as shown 
in Figure 3. We adjusted the parameters of the competing methods to elicit their best performance. The reconstruction results show that the proposed CRDN works better than the three comparison methods. The CRDN removes artifacts better and retains more details, especially the details marked by the red arrow. We also show the quantitative evaluations of the CRDN and the three comparison models in Table 1. One can see that the CRDN achieves better quantitative evaluations than do the other models. So the CRDN is superior to the $L+S$, the D5C5 model, and the DIMENSION model in both visual results and quantitative indicators, which indicates the efficacy of the CRDN.

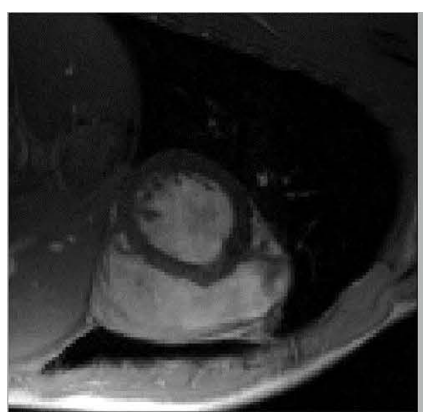

(a) ground truth

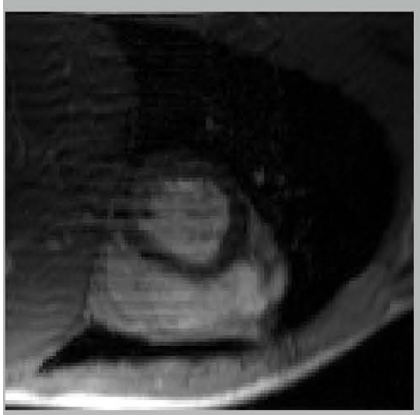

(d) L+S

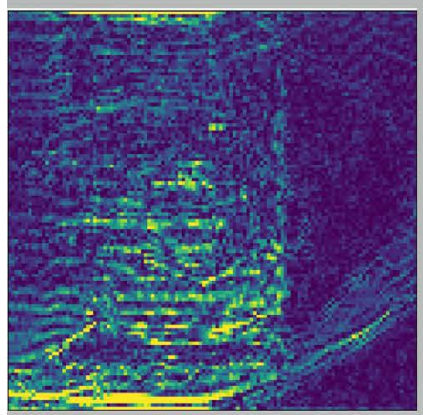

(h) L+S (error)

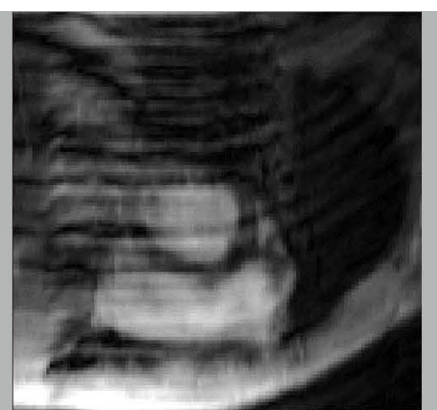

(b) zero filling

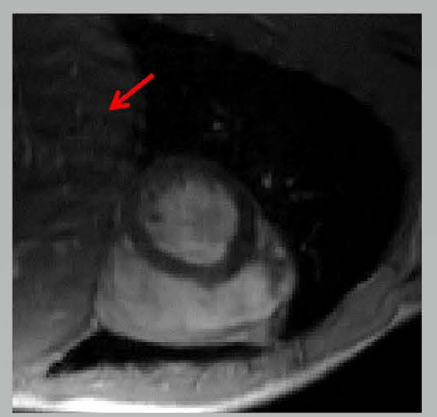

(e) $\mathrm{D} 5 \mathrm{C} 5$

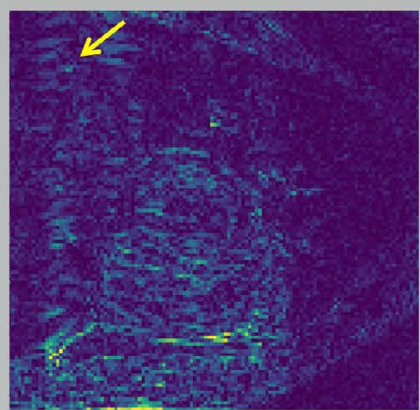

(i) D5C5 (error)

b. Does the Total Variation Loss Function Work?

To demonstrate the efficacy of the total variation loss

Table 1. The Average MSE, PSNR, and SSIM of Zero-Filling, L+S, D5C5, DIMENSION, and CRDN on the Test Dataset

\begin{tabular}{lccc} 
& MSE & PSNR & SSIM \\
Zero filling & 0.004724 & 23.2146 & 0.7637 \\
\hline L+S & 0.000737 & 26.5413 & 0.8688 \\
\hline D5C5 & 0.000301 & 35.1694 & 0.9775 \\
\hline DIMENSION & 0.000173 & 37.5856 & 0.9846 \\
\hline CRDN & 0.000158 & 37.9785 & 0.9857
\end{tabular}

CRDN = cascaded residual dense networks; MSE = mean square error; PSNR = peak signal-to-noise ratio; SSIM = structural similarity index

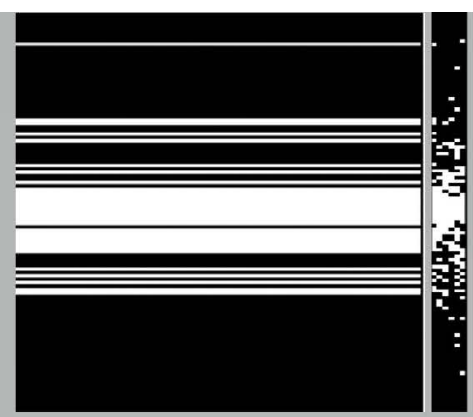

(c) mask

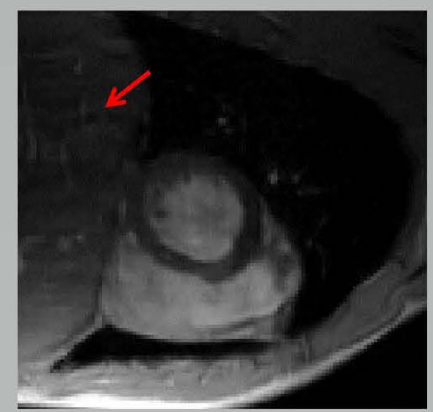

(f) DIMENSION

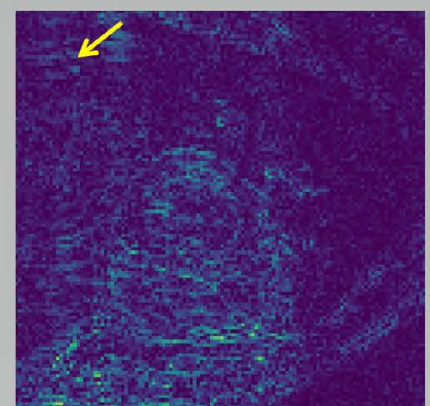

(j) DIMENSION (error)

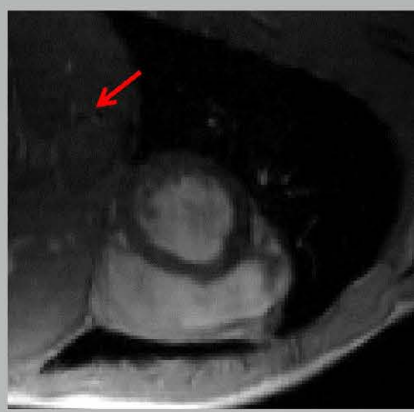

(g) CRDN

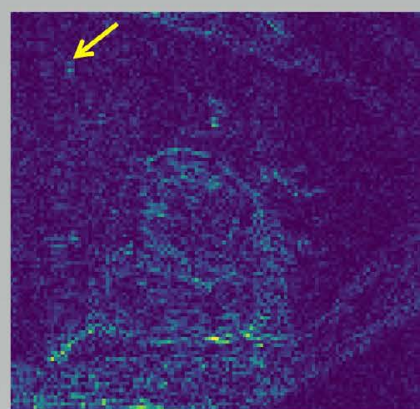

(k) CRDN (error)

Fig. 3. The reconstructions of the $L+S, D 5 C 5$, DIMENSION, and the proposed CRDN. (a) ground truth, (b) zero-filling, (c) mask and its $k$ - $t$ extraction, $(d)$ the $L+S$ reconstruction, (e) the D5C5 reconstruction, $(f)$ the DIMENSION, $(g)$ the CRDN reconstruction, $(h-k)$ their corresponding error maps with display ranges $(0,0.07)$. 
function, we compared different CRDN models with different types of TV, as shown in Figure 4. We found that the reconstruction results of the models with the TV loss function were improved more than were those without. The quantitative indicators in Table 2 also have consistent

Table 2. The Average MSE, PSNR, and SSIM of Different Loss Functions on the Test Dataset

\begin{tabular}{lccc} 
& MSE & PSNR & SSIM \\
Zero filling & 0.003578 & 24.0981 & 0.8242 \\
\hline MSE & 0.000249 & 35.6752 & 0.9728 \\
\hline TV-aniso & 0.000237 & 35.8934 & 0.9731 \\
\hline TV-iso & 0.000245 & 35.7388 & 0.9723 \\
\hline 2DTV & 0.000246 & 35.7185 & 0.9723 \\
\hline 3DTV & 0.000241 & 35.8062 & 0.9724
\end{tabular}

$2 \mathrm{DTV}=2$-degree TV; $3 \mathrm{DTV}=3$-degree TV; MSE = mean square error; PSNR = peak signal-to-noise ratio; SSIM = structural similarity index; TV = total variation; TV-aniso $=$ TV-anisotropic; TV-iso $=$ TV-isotropic conclusions. Different TV loss functions had different degrees of improvement in the results, in which TV-aniso has the optimal improvement effect.

\section{CONCLUSION AND OUTLOOK}

Our previous work proposed a dynamic MR imaging method with both $k$-space and spatial prior knowledge integrated via multi-supervised network training. Nevertheless, there was still some smoothing needed in the reconstructed images at high acceleration factors. In this work, we propose cascaded residual dense networks for dynamic MR imaging with edge-enhanced loss constraint, dubbed CRDN. Specifically, the cascaded residual dense networks fully exploit the hierarchical features from all the convolutional layers with both local and global feature fusion. We further use the TV loss function, which has the edge enhancement properties, for training the networks.

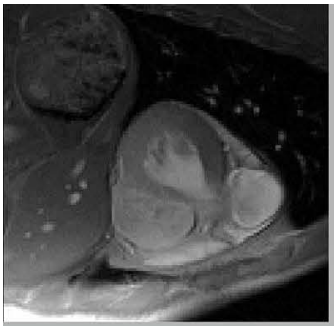

(a) ground truth

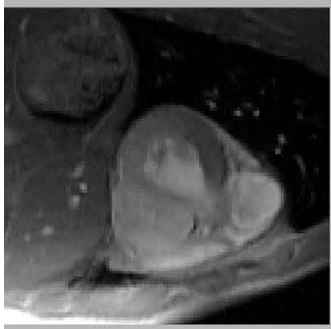

(d) MSE

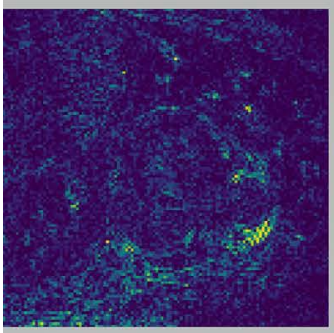

(i) MSE (error)

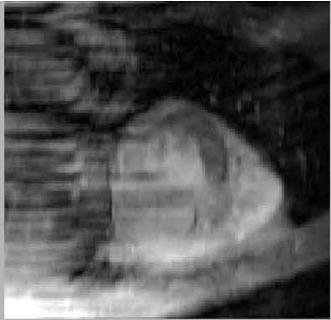

(b) zero filling

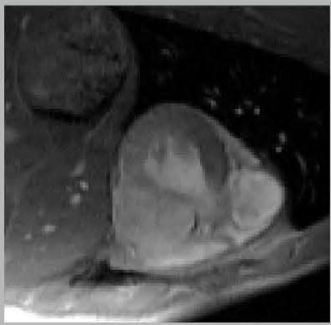

(e) TV-aniso

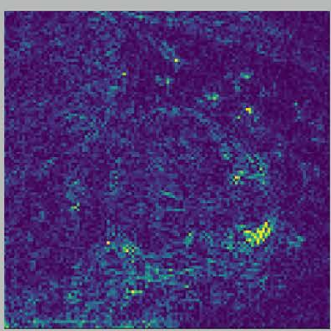

(j) TV-aniso (error)

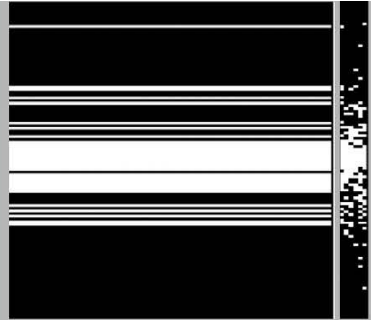

(c) mask

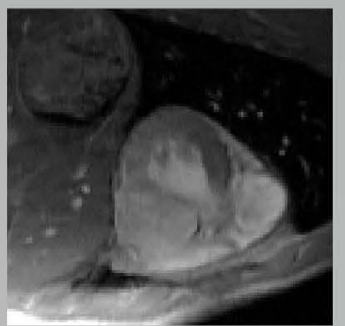

(f) TV-iso

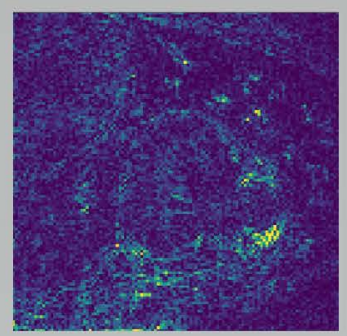

(k) TV-iso (error)

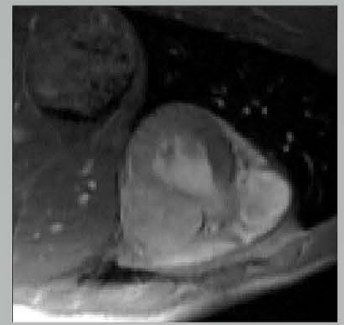

(g) 2DTV

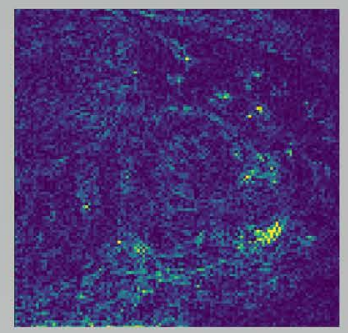

(I) 2DTV (error)

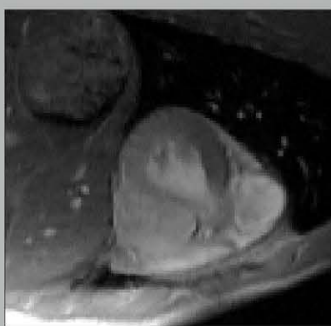

(h) 3DTV

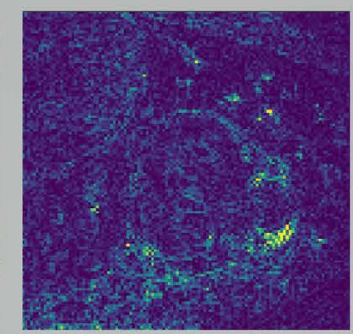

(m) 3DTV (error)

Fig. 4. The reconstructions of different types of TV loss functions at 4-fold acceleration. (a) ground truth, (b) zero-filling, (c) mask and its k-t extraction, (d) the MSE loss, (e) the TV-aniso loss, $(\mathrm{f})$ the TV-iso loss, $(\mathrm{g})$ 2DTV loss, (h) 3DTV loss, (i-m) their corresponding error maps with display ranges $(0,0.07)$. 


\section{Acknowledgments}

This research was partly supported by the National Natural Science Foundation of China $(61771463,81830056$, U1805261, 81971611, 61871373, 81729003, 81901736); National Key R\&D Program of China (2017YFC0108802 and 2017YFC0112903); Natural Science Foundation of Guangdong Province (2018A0303130132); Shenzhen Key Laboratory of Ultrasound Imaging and Therapy (ZDSYS20180206180631473); Shenzhen Peacock Plan Team Program (KOTD20180413181834876); Innovation and Technology Commission of the government of Hong Kong SAR (MRP/001/18X); Strategic Priority Research Program of the Chinese Academy of Sciences (XDB25000000).

\section{REFERENCES}

1. Kaiser WA, Zeitler E. MR imaging of the breast: fast imaging sequences with and without Gd-DTPA. Preliminary observations. Radiology 1989;170:681-686

2. Sodickson DK, Manning WJ. Simultaneous acquisition of spatial harmonics (SMASH): fast imaging with radiofrequency coil arrays. Magn Reson Med 1997;38:591603

3. Donoho DL. Compressed sensing. IEEE Trans Inf Theory 2006;52:1289-1306

4. Lustig M, Donoho D, Pauly JM. Sparse MRI: the application of compressed sensing for rapid MR imaging. Magn Reson Med 2007;58:1182-1195

5. Jung $H_{1}$ Ye JC, Kim EY. Improved k-t BLAST and k-t SENSE using FOCUSS. Phys Med Biol 2007;52:3201-3226

6. Tsao J, Boesiger P, Pruessmann KP. k-t BLAST and k-t SENSE: dynamic MRI with high frame rate exploiting spatiotemporal correlations. Magn Reson Med 2003;50:1031-1042

7. Liang D, DiBella EV, Chen RR, Ying L. k-t ISD: dynamic cardiac MR imaging using compressed sensing with iterative support detection. Magn Reson Med 2012;68:4153

8. Caballero J, Price AN, Rueckert D, Hajnal JV. Dictionary learning and time sparsity for dynamic MR data reconstruction. IEEE Trans Med Imaging 2014;33:979-994

9. Wang Y, Ying L. Compressed sensing dynamic cardiac cine MRI using learned spatiotemporal dictionary. IEEE Trans Biomed Eng 2014;61:1109-1120

10. Otazo R, Candes E, Sodickson DK. Low-rank plus sparse matrix decomposition for accelerated dynamic MRI with separation of background and dynamic components. Magn Reson Med 2015;73:1125-1136
11. Lingala SG, Hu Y, DiBella E, Jacob M. Accelerated dynamic MRI exploiting sparsity and low-rank structure: k-t SLR. IEEE Trans Med Imaging 2011;30:1042-1054

12. Yang $Y$, Sun J, Li $H, X u$ Z. Deep ADMM-net for compressive sensing MRI. Advances in Neural Information Processing Systems (NIPS), 2016

13. Hammernik K, Klatzer $T$, Kobler $E$, et al. Learning a variational network for reconstruction of accelerated MRI data. Magn Reson Med 2018;79:3055-3071

14. Knoll F, Hammernik K, Kobler E, Pock T, Recht MP, Sodickson DK. Assessment of the generalization of learned image reconstruction and the potential for transfer learning. Magn Reson Med 2019;81:116-128

15. Wang S, Su Z, Ying $L$, et al. Accelerating magnetic resonance imaging via deep learning. Proc IEEE Int Symp Biomed Imaging 2016:514-517

16. Kwon K, Kim D, Park H. A parallel MR imaging method using multilayer perceptron. Med Phys 2017;44:6209-6224

17. Han Y, Yoo J, Kim HH, Shin HJ, Sung K, Ye JC. Deep learning with domain adaptation for accelerated projectionreconstruction MR. Magn Reson Med 2018;80:1189-1205

18. Zhu B, Liu JZ, Cauley SF, Rosen BR, Rosen MS. Image reconstruction by domain-transform manifold learning. Nature 2018;555:487-492

19. Eo T, Jun Y, Kim T, Jang J, Lee HJ, Hwang D. KIKI-net: crossdomain convolutional neural networks for reconstructing undersampled magnetic resonance images. Magn Reson Med 2018;80:2188-2201

20. Sun L, Fan $Z$, Huang $Y$, Ding $X$, Paisley J. Compressed sensing MRI using a recursive dilated network. AAAI 2018;2444-2451

21. Quan TM, Nguyen-Duc T, Jeong WK. Compressed sensing MRI reconstruction using a generative adversarial network with a cyclic loss. IEEE Trans Med Imaging 2018;37:14881497

22. Schlemper J, Caballero J, Hajnal JV, Price AN, Rueckert D. A deep cascade of convolutional neural networks for dynamic MR image reconstruction. IEEE Trans Med Imaging 2018;37:491-503

23. Wang $S$, Ke $Z$, Cheng $H$, et al. DIMENSION: dynamic MR imaging with both k-space and spatial prior knowledge obtained via multi-supervised network training. NMR Biomed 2019;4:e4131

24. Qin C, Schlemper J, Caballero J, Price AN, Hajnal JV, Rueckert D. Convolutional recurrent neural networks for dynamic MR image reconstruction. IEEE Trans Med Imaging 2019;38:280-290

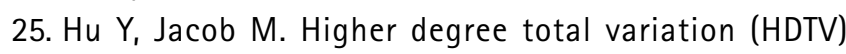
regularization for image recovery. IEEE Trans Image Process 2012;21:2559-2571 
26. Walsh DO, Gmitro AF, Marcellin MW. Adaptive reconstruction of phased array MR imagery. Magn Reson Med 2000;43:682-690

27. He K, Zhang $X$, Ren $S$, Sun J. Deep residual learning for image recognition. Computer Vision and Pattern Recognition (CVPR), IEEE 2016:770-778

28. LeCun $Y$, Bengio $Y$, Hinton G. Deep learning. Nature 2015;521:436-444

29. He K, Zhang $X$, Ren $S$, Sun J. Delving deep into rectifiers: surpassing human-level performance on ImageNet classification. International Conference on Computer Vision (ICCV), IEEE 2015:1026-1034
30. Glorot X, Bordes A, Bengio Y. Deep sparse rectifier neural networks. AISTATS 2011;315-323

31. Zeiler MD. ADADELTA: an adaptive learning rate method. arXiv preprint arXiv:1212.5701, 2012

32. Kingma DP, Ba JL. Adam: a method for stochastic optimization. arXiv preprint arXiv:1412.6980, 2014

33. Abadi M, Barham P, Chen J, et al. Tensorflow: a system for large-scale machine learning. OSDI 2016;16:265-283

34. Wang Z, Bovik AC, Sheikh HR, Simoncelli EP. Image quality assessment: from error visibility to structural similarity. IEEE Trans Image Process 2004;13:600-612 